limitations of the current decision-making process. We propose some modifications in approach to determination of Specific Causation that may better address issues of multiple additive or interacting causal factors, acceleration of phenotypic expression of a disease, aggravation of pre-existing disease, and challenges of applying medicolegal criteria that do not account for these factors.

Discussion We discuss alternative approaches to Specific Causation that incorporate recent scientific developments in causal inference, explicitly address some of the existing inadequacies, and aim to enable more fair and accurate decision-making with respect to occupational disease causation in individuals.

\section{CHRONIC VENOUS DISEASE IN OCCUPATIONAL ENVIRONMENT: WHICH AXES OF PREVENTION?}

${ }^{1,2,3}$ Faiza Bossy. ${ }^{1}$ Horizon Sante Travail, Paris, France; ${ }^{2}$ Ultrasongraphy, Paris, France;
${ }^{3}$ Institute Franco Britanique Emergency, Levallois Perret, France

\subsection{6/oemed-2018-ICOHabstracts.1049}

Objectives The venous disease represents a major problem of public health, it is responsible for absenteeism and for hospitalisation. It has an indirect impact on productivity. Nevertheless it probably remains underestimated. The purpose of this epidemiological study is to identify the risk factors of the venous disease and in particular to assess the state of the art on the link between professional postures and the occurrence of disease.

Methods This project is a critical reading. It relies on three sources of data: scientific articles, grey literature and one academic book. The strategy of document retrieval and the analysis of the literature rests on an evaluation guide published by the ANAES.

Results The venous disease is a chronic pathology. The venous disease is multifactorial. Age is the most significant risk factor among all examined components: sex, heredity, and pregnancy. Socio-environmental factors favour the onset of the disease. About the working situations, the prolonged exposure to static standing positions and the trampling are perceived as more constraining than sitting position. Lifting heavy load, warm occupational surrounding and tight clothes increase the complaints of the employees in static position. Alternating different working positions and walking are protective factors of the chronic venous disease.

Conclusion The chronology of the studies spans nearly 50 years. The nature of the work environment, in particular of working conditions, is evolving. Nowadays the static position is more often sitting. The international specific venous disease classification recently created, is widely used by professionals.

This study confirms strong prevalence of the venous disease generally and in particular in occupational environment.

The results contain a clear interest allowing to develop axes of prevention of the venous disease at work.

\section{MEDICAL ASPECTS OF CONTRACTOR GOVERNANCE - AN OIL AND GAS INDUSTRY'S PERSPECTIVE}

Syed Zaffar Hussaini*. Specialist in Occupational Medicine RaGas Company Limited, Doha, State of Qatar

10.1136/oemed-2018-ICOHabstracts. 1050
Introduction The Contractor workforce forms an integral part of any industry. It is necessary to ensure that the Contract workers are Fit to do their job as this would enable companies to minimise the risk of an adverse consequence to the health and/or safety of an employee resulting from a foreseeable health condition.

Methods A cross sectional study was carried out to review the validation and audit of Fitness to work assessments among Contactors working in the company on onshore and offshore locations in order to determine 'key learning related to Medical aspects of Contractor Governance. The data included Fitness assessments conducted between 2011 to 2017.

Results

- Elements of Medical 'Fitness for work' expectations were integrated in the planning stages (into contactor procurement and Contractual agreements).

- The ownership of Contractor employees' health should be the responsibility of the Contractors' company and the contractors should take reasonable care of their own health and safety.

- The benefits of validation and audit of Fitness to work assessments/Safety Critical Task Assessments and successful management of contractor employees with known chronic medical conditions.

- Oil and Gas companies should maintain minimum standards for Offshore Medical Fitness and align with Oil and Gas Industry's best practice approach and make consistent decisions in accordance with stipulated standards.

- The proven advantages of Offshore Medical Fitness cards for employees who are expected to travel and work offshore.

This has been a cost effective initiative since it has reduced costs of unnecessary Medical evacuations.

Discussion This study establishes the advantages of better management of Medical Aspects of Contractor Governance which focuses on Contractor employees' health issues related to existing medical conditions and work-life balance which would enable companies to achieve the goal of a 'Safe, Healthy, Happy and Fit workforce'.

\section{IS DRINKING ALCOHOL REALLY USEFUL FOR RELEASING STRESS?}

${ }^{1}$ Hiroyuki Hibino, ${ }^{1}$ Hiroshi Tanaka, ${ }^{1}$ Taisuke Tomonaga, ${ }^{2}$ Seichi Horie. ${ }^{1}$ Nippon Steel and Sumitomo Metal Corporation; ${ }^{2}$ University of Occupational and Environmental Health, Japan

\subsection{6/oemed-2018-ICOHabstracts. 1051}

Introduction Workers often drink alcohol for releasing stress. However, many reports show that heavy drinking is a risk of depression. Some mentioned the influence of not only the amount of drinking alcohol but also the wrong way of using alcohol like binge drinking should be examined. Alcohol Use Disorders Identification Test (AUDIT) can evaluate the way of one's using alcohol and the amount of drinking. Thus it is able to examine the more accurate influence of alcohol for depression.

In this study, we analysed the relationship between AUDIT score and depression from work-related stress by The Brief Job Stress Questionnaire (BJSQ).

Methods 4799 workers in a manufacturing company participated in this study. We analysed the data obtained from 4709 male employees without any missing value in logistic analysis and categorised depression as the dependent variable (13:high 
score group, $12 \geqq$ : low score group), AUDIT score (0-14: group I, 15-19: group II, 20-: group III), job status (managerial or not), overtime work (per month, $<40$ hours: mild, 40-80 hours: middle, $>80$ hours: severe), quantitative overload (high/middle/low), qualitative overload (high/middle/low), stress with interpersonal relationships (high/middle/low), and job control (high/middle/low), as independent variables.

Results Depression tended to be higher in group II of AUDIT (OR: $1.40,95 \% \mathrm{CI}: 0.99$ to $1.96, \mathrm{p}=0.54$ ) and group III (OR: 2.45 , 95\% CI: 1.36 to $4.42, \mathrm{p}=0.003$ ). About overtimework, the severe group was more depressive than mild group (OR: $2.38,95 \% \mathrm{CI}: 1.45$ to $3.90, \mathrm{p}<0.001)$. More qualitative overload caused stronger depression (OR: 2.45, 95\% CI: 1.43 to $4.22, \mathrm{p}<0.001)$. Stress with interpersonal relationships also was related to depression (OR: 9.49, 95\% CI: 4.94 to 18.22, $\mathrm{p}<0.001)$.

Conclusion Higher AUDIT score seemed to show higher risk of developing depression. The wrong way of using alcohol may be an exacerbating factor of depression. We should be careful to drinking on the purpose of releasing stress.

\section{ASSOCIATION BETWEEN JOB STRESS AND OCCUPATIONAL INJURIES AMONG KOREAN FIREFIGHTERS: A NATIONWIDE CROSS-SECTIONAL STUDY}

\begin{abstract}
${ }^{1,2}$ Yeong-Kwang Kim, ${ }^{3}$ Yeon-Soon Ahn, ${ }^{4}$ Kyoo-Sang Kim, ${ }^{1,2,5,6} \mathrm{Jin}-\mathrm{Ha}$ Yoon, 1,2,5,6Jaehoon Roh*. 'Graduate School of Public Health, Yonsei University, South Korea, Seoul; ${ }^{2}$ The Institute for Occupational Health, Yonsei University College of Medicine, South Korea, Seoul; ${ }^{3}$ Department of Occupational and Environmental Medicine, Dongguk University Ilsan Hospital, South Korea, Ilsan; ${ }^{4}$ Department of Occupational and Environmental Medicine, Seoul Medical Centre, South Korea, Seoul; ${ }^{5}$ Department of Preventive Medicine, Yonsei University College of Medicine, South Korea, Seoul; ${ }^{6}$ Incheon Workers' Health Centre, South Korea, Incheon
\end{abstract}

\subsection{6/oemed-2018-ICOHabstracts. 1052}

Introduction Firefighters are responsible for the safety of citizens, and perform functions that include fire suppression and emergency medical services (EMS). As such, they are exposed to physical or chemical hazards that lead to high rates of occupational injuries. Despite a plethora of studies, there have been only a few systematic investigations to identify factors influencing occupational injuries among firefighters. In this study, which is based on a survey of all Korean firefighters, we aimed to investigate the existence of a correlation between job stress and occupational injury among firefighters.

Methods Cross-sectional study was conducted via a survey targeting firefighters in South Korea between July and November 2007. A questionnaire was mailed to 30630 firefighters; $25615(83.6 \%)$ responded. Statistical analysis was performed using the SAS 9.2 software (SAS Institute Inc., Cary, NC, USA).

Results Among fire suppression personnel, high job demands $(\mathrm{OR}=1.49,95 \% \mathrm{CI}: 1.25$ to 1.77$)$, high interpersonal conflicts $(\mathrm{OR}=1.18,95 \% \mathrm{CI}: 1.02$ to 1.37$)$, a poor organisational system $(\mathrm{OR}=1.33$, 95\% CI: 1.14 to 1.55$)$, and a negative workplace environment $(\mathrm{OR}=1.41,95 \% \mathrm{CI}: 1.21$ to 1.64$)$, and high job demands $(\mathrm{OR}=1.22,95 \% \mathrm{CI}$ : 1.01 to 1.47$)$ were associated with the occurrence of occupational injury. Among emergency medical services personnel, high job demands $(\mathrm{OR}=1.26,95 \% \mathrm{CI}: 1.03$ to 1.54$)$, high interpersonal conflicts $(\mathrm{OR}=1.40,95 \% \mathrm{CI}: 1.19$ to 1.66$)$, a poor organisational system $(\mathrm{OR}=1.55,95 \% \mathrm{CI}: 1.30$ to 1.85$)$, lack of reward $(\mathrm{OR}=1.43,95 \% \mathrm{CI}: 1.21$ to 1.69$)$ and a negative workplace environment $(\mathrm{OR}=1.30,95 \% \mathrm{CI}: 1.10$ to 1.54$)$ were associated with the occurrence of occupational injury.

Conclusion High job stress among firefighters was associated with both the occurrence of occupational injury, and also with an increased frequency of injuries. Therefore, job stress should be addressed to prevent occupational injuries among firefighters.

\section{DIFFERENCES BETWEEN PERITONEAL AND PLEURAL MESOTHELIOMA IN LOMBARDY, ITALY}

${ }^{1} \mathrm{D}$ Consonni*, ${ }^{2} \mathrm{M}$ Mendola, ${ }^{1} \mathrm{~B}$ Dallari, ${ }^{2} \mathrm{M}$ Sokooti, ${ }^{3} \mathrm{R}$ Tabibi, ${ }^{2} \mathrm{R}$ Callegari, ${ }^{4} \mathrm{~L}$ Riboldi, ${ }^{1} \mathrm{C}$ Mensi. 'Epidemiology Unit, Fondazione IRCCS Ca' Granda Ospedale Maggiore Policlinico, Milan, Italy, ${ }^{2}$ Università degli Studi di Milano, Milan, Italy; ${ }^{3}$ Abadan School of Medical Sciences, Abadan, Iran; ${ }^{4}$ Workers Health Protection and Promotion Unit, Fondazione IRCCS Ca' Granda Ospedale Maggiore Policlinico, Milan, Italy

\subsection{6/oemed-2018-ICOHabstracts. 1053}

Introduction Asbestos is the main risk factor for development of both peritoneal (PEM) and pleural (PLM) mesothelioma, but characteristics of this two diseases and their association with asbestos show some differences. We examined characteristics of PEM and PLM in Lombardy, Italy, the most populated (currently, 10 million residents) and industrialised Italian region.

Methods The RML, established in 2000, is a population-based registry which records all cases of mesothelioma among Lombardy residents. It is part of a national network of mesothelioma registries (ReNaM). Notification of mesothelioma cases is compulsory by law. Additional sources of mesothelioma cases include hospital admission, mortality, occupational diseases, and pathology databases. Verified mesothelioma cases are interviewed about past asbestos exposure using a standardised questionnaire. For this study, from the RML database we selected PEM and PLM cases diagnosed in 2000-2014. We investigated asbestos exposure, presence of asbestosis or pleural plaques, and past employment in economical sectors.

Results We identified 300 PEM and 5,011 PLM cases. Incidence rates (per 1.000.000 person-years, world standardised) of PEM were 2.4 (men) and 1.7 (women), compared with 52.4 and 19.9 for PLM. Asbestosis (both genders) and pleural plaques (men) were more frequent among PEM cases. Occupational asbestos exposure was similar in PEM and PLM cases. We found higher proportions of PEM employed in the asbestos cement production.

Conclusion The higher frequency of pleural plaques in PEM cases and the similar frequency of past occupational asbestos exposure confirm the association between asbestos exposure and peritoneal mesothelioma. The higher proportions of asbestosis and of past employment in the asbestos-cement sector among PEM cases suggest a possible role of high exposures to asbestos in the peritoneal mesothelioma genesis. 